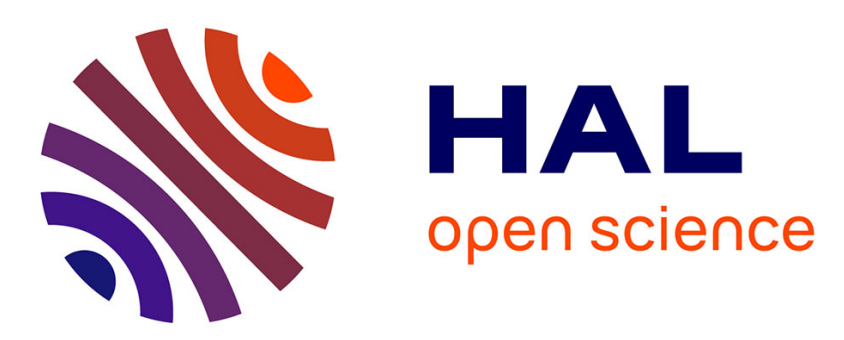

\title{
Adhesion forces controlled by chemical self-assembly and pH. Application to robotic microhandling.
}

Jérôme Dejeu, Michaël Gauthier, Patrick Rougeot, Wilfrid Boireau

\section{To cite this version:}

Jérôme Dejeu, Michaël Gauthier, Patrick Rougeot, Wilfrid Boireau. Adhesion forces controlled by chemical self-assembly and $\mathrm{pH}$. Application to robotic microhandling.. Applied Material \& Interfaces, 2009, 1 (9), pp.1966-1977. 10.1021/am900343w . hal-00413803

\section{HAL Id: hal-00413803 https://hal.science/hal-00413803}

Submitted on 7 Sep 2009

HAL is a multi-disciplinary open access archive for the deposit and dissemination of scientific research documents, whether they are published or not. The documents may come from teaching and research institutions in France or abroad, or from public or private research centers.
L'archive ouverte pluridisciplinaire HAL, est destinée au dépôt et à la diffusion de documents scientifiques de niveau recherche, publiés ou non, émanant des établissements d'enseignement et de recherche français ou étrangers, des laboratoires publics ou privés. 


\title{
Adhesion forces controlled by chemical self-assembly and $\mathrm{pH}$, application to robotic microhandling
}

\author{
Jérôme Dejeu, Michaël Gauthier, ${ }^{*}$ Patrick Rougeot, and Wilfrid Boireau \\ FEMTO-ST Institute, UMR CNRS 6174 - UFC / ENSMM / UTBM, 24 rue Alain Savary, 25000 \\ Besançon, France
}

\begin{abstract}
Robotic microhandling is a promising way to assemble microcomponents in order to manufacture new generation of Hybrid Micro ElectroMechanical Systems (HMEMS). However, at the scale of several micrometers, adhesion phenomenon highly perturbs the micro-objects release and the positioning. This phenomenon is directly linked to both the object and the gripper surface chemical composition. We propose to control adhesion by using chemical selfassembly monolayer (SAM) on both surfaces. Different types of chemical functionalisation have been tested and this paper focuses on the presentation of aminosilane grafted (3 (ethoxydimethylsilyl) propyl amine (APTES) and (3 aminopropyl) triethoxysilane (APDMES)). We show that the liquid $\mathrm{pH}$ can be used to modify the adhesion and to switch from an attractive behaviour to a repulsive behaviour. The $\mathrm{pH}$ control can thus be used to increase adhesion during handling and cancel adhesion during release. Experiments have shown that the $\mathrm{pH}$ control is able to control the release of a micro-object. This paper shows the relevance of a new type of reliable submerged robotic microhandling principle, which is based on adjusting chemical properties of liquid.
\end{abstract}




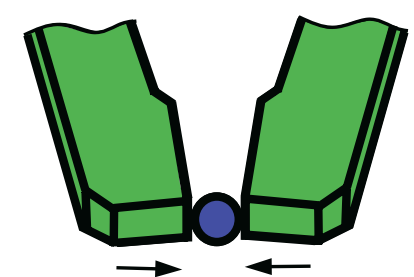

(Grasping pH5.5)

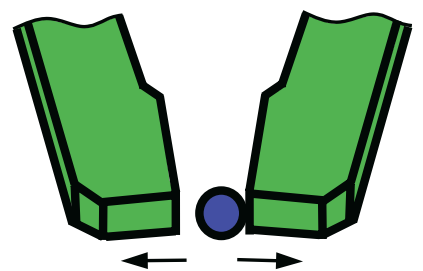

(Release pH5.5)

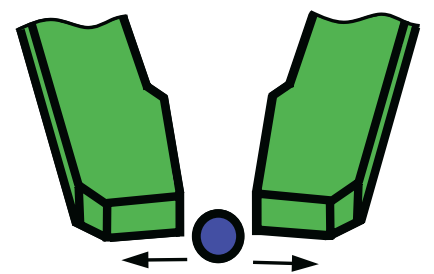

(Release pH9)
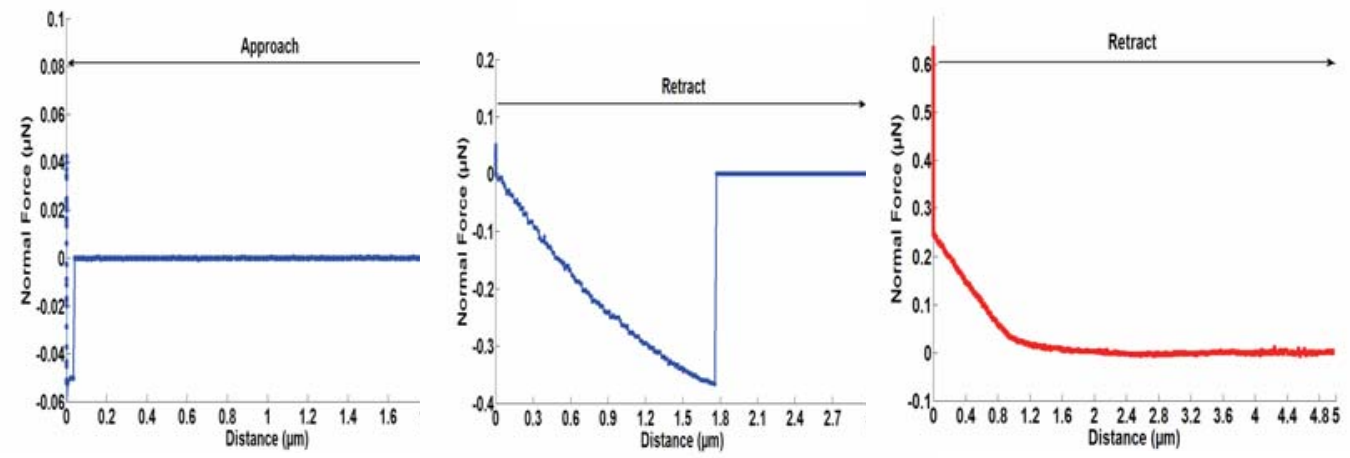

KeyWord: Microhandling, Surface functionalisation, Aminosilane, Adhesion, Pull-off forces

\section{Introduction}

Manufactured products are getting smaller and smaller and are integrating more and more functionalities in small volumes. Several application fields are concerned such as telephony, bioengineering, telecommunications or more generally speaking the Micro-Electro-Mechanical-Systems (MEMS). The assembly of these microproducts is a great challenge because of the microscopic size of the components. ${ }^{1}$ In fact, the major difficulty of micro-assembly comes from the particularity of the micro-objects behaviour which depends on surface forces. ${ }^{2-4}$ The magnitude of the physical effects is drastically modified with the changing scale from macroscopic scale ( $1 \mathrm{~mm}$ for example) to the microscopic scale ( $1 \mu \mathrm{m}$ for example), the lengths are divided by $10^{3}$, the weight is divided by $10^{9}$ while the surface forces (e.g. van der Waals force) are divided only by $10^{6}$. The effect of the gravity thus decreases more rapidly than the effect of surface forces during miniaturisation. So, in 
microscale, the surface forces are predominant compare to the weight and the objects tends to stick the microgrippers. The manipulation of a micro-object requires handling, positioning, and releasing it without disturbances of the surface forces such as electrostatic forces, van der Waals forces or capillary forces. The release is the most critical phase which is usually hindered by adhesion.

Several methods have been proposed in the last ten years to improve micromanipulation. ${ }^{5,6}$ The first approach consists in using non-contact manipulation like laser trapping ${ }^{7}$ or dielectrophoresis. ${ }^{8}$ These manipulation methods are not disturbed by adhesion. However, in an applicative context, micromanipulation has to be able to induce large force (e.g. several $\mu \mathrm{N}$ ) without displacement in order to assemble objects (e.g. insertion). In non-contact manipulation, the blocking force which represents the maximal force applied on an object without displacement stays low which is a major drawback in the application field of microassembly. The second approach deals with contact manipulation where the adhesion is reduced or directly used for manipulation. The reduction of the adhesion can be achieved for example by raising the roughness of the end-effectors. ${ }^{9,10}$ Adhesion can be directly used to perform manipulation tasks. In this case, one-fingered gripper is sufficient to handle objects, but releasing stays difficult. ${ }^{11}$ In fact, new release methods are required such as inertial ${ }^{12}$ or dielectrophoresis release. ${ }^{13}$ The major advantage of contact handling consists in the fact that the blocking force is usually high. The current microhandling methods are able to improve micromanipulation but the object behaviour is always disturbed by adhesion and the reliability is still low. ${ }^{10,14}$ The knowledge of adhesion forces is thus essential to enable the advent of reliable micromanipulation techniques. Current approaches are based on experimental measurements performed with atomic force microscopes (AFM), ${ }^{15-18}$ interferometric surface force apparatus, ${ }^{19,20}$ capacitive force sensors, ${ }^{21}$ nanoindentation testers, ${ }^{22,23}$ tangential streaming potential, ${ }^{24}$ or a measurement platform utilizing the contact mechanics theory of Johnson, Kendall,and Roberts (JKR). ${ }^{25}$ The adhesion force measurement is influenced by several parameters such as preload force, ${ }^{26,27}$ humidity, ${ }^{28}$ temperature,${ }^{29}$ pressure,${ }^{30}$ roughness, ${ }^{31}$ properties of the liquid medium ( $\mathrm{pH}^{32,33}$ and ionic strength $\left.{ }^{32,34}\right)$.

We propose a new contact handling system that chemically controls the surface forces between 
the object and the gripper. ${ }^{15}$ We have already shown that the medium (air or liquid) can modify or cancel the adhesion force. ${ }^{15}$ The major objective is to control the adhesion force or to create a repulsive force to guarantee a reliable release. Now, the surface properties of a material can be controlled by surface functionalisation in a liquid. The surface functionalisation of the objects or of the grippers can be obtained by different methods. The two most important methods to form self-assembled monolayers are the polyelectrolyte physisorption (polyelectrolyte with positive or negative charges) ${ }^{35}$ and the surface molecules grafting (covalent bond between the substrate and the molecules). ${ }^{36-38}$ This phenomenon is mainly due to two processes depending of the instrinsic atomic compositions of the substrates: oxidized substrates are useful for silane functionalisation whereas gold substrates are only reactive with thiol compounds. The difference in reactivity could be a real advantage in our approach because the using of silane (or thiol) molecules will allow a vectorization of the chemical processes. This could be a highly versatile tool box to give specific properties to a precise part of the micro-objects especially in micro-assembly.

The grafting generates covalent bond between substrate and molecules. These molecules must contain silanol, thiol, azide, allyl or vinyl groups ${ }^{36,37}$ in one extremity. These molecules have to be used in organic solvent such as toluene, acetone, methanol, ethanol, ... The silanol create a Si-O-Si bond with the silica substrate ${ }^{36}$ while allyl or vinyl generates $\mathrm{Si}-\mathrm{O}-\mathrm{C}$ (or Si-C) bond ${ }^{39}$ and the azide groups produce $\mathrm{Si}-\mathrm{N}$ bond. ${ }^{40}$ In this work, we choose the silanisation because the layer created did not exhibit any signature of degradation when stored in an airtight container for 18 months, ${ }^{41}$ was stable up to a temperature near of $350^{\circ} \mathrm{C},{ }^{42,43}$ even when washed using $1 \%$ detergent solution, hot tap water or organic solvents and aqueous acid at room temperature. ${ }^{42}$ This silane layer was robust under the same daunting conditions that all existing semiconductor materials already endure such as thermal stability up to $350^{\circ} \mathrm{C}$, chemical stability under different etchants. So the functionalised MEMS can be used in molecular and/or hybrid electronics. The charge density of functionalised surfaces must depend on the $\mathrm{pH}$ in order to control the adhesion force in liquid medium using the $\mathrm{pH}$. 
The microhandling principle is presented in Figure 1. The grasping can be done at $\mathrm{pH}_{1}$ where the surface charges on the gripper and the object induces an attractive force. In order to release the object, the $\mathrm{pH}$ is modified to a second value $\mathrm{pH}_{2}$ where the object charge is changing. The electrostatic force becomes repulsive and the object is released.

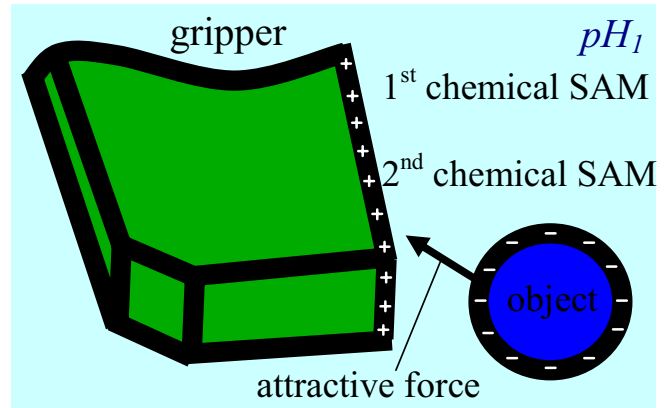

(a) handling in $\mathrm{pH}_{1}$ where charges in SAM induces attractive force

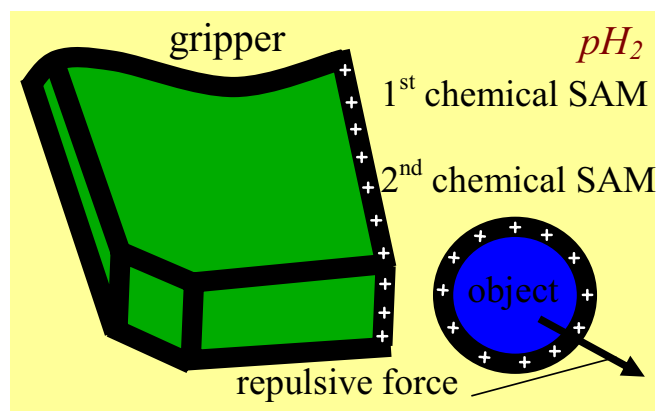

(b) release in $\mathrm{pH}_{2}$ where charges in SAM induces repulsive force

Figure 1: Principle of the Robotic Microhandling controlled by Chemical Self Assembly Monolayer (SAM)

The microhandling method proposed is based on two chemical functions: amine and silica. In one hand, the amine group is in state $\mathrm{NH}_{2}$ in basic $\mathrm{pH}$ and in $\mathrm{NH}_{3}^{+}$in acidic $\mathrm{pH}$. In the other hand, the silica surface charge in water is naturally negative excepted for very acidic $\mathrm{pH}$ where the surface is weakly positive. ${ }^{44}$

The objective of this article consists in showing the relevance of $\mathrm{pH}$ switching to control submerged microhandling. In the preliminary section, we presented the surface chemical functionalizations. In the second part, we analysed the interaction force measurement between two surfaces (functionalised and not functionalised or both functionnalised) in liquid in function of the $\mathrm{pH}$ in order to determine the charge density of the silane layer by a model of the surface charges. Finally, we deal with an experimental micromanipulation task between a glass sphere and an AFM tipless controled by the $\mathrm{pH}$ solution. 


\section{Experimental details}

\section{Materials and chemicals}

Two silane functionalisations have been tested (see in Figure 2): the 3 (ethoxydimethylsilyl) propyl amine (APTES) and the silane, (3 aminopropyl) triethoxysilane (APDMES). Both chemical compounds (APTES, APDMES) used to surface functionalisation are amine functions $\mathrm{NH}_{2}$ which can be protonated or ionised to $\mathrm{NH}_{3}^{+}$according to $\mathrm{pH}$. In acidic $\mathrm{pH}$, the amine is totally ionised, then the ionisation decreases and is null in basic $\mathrm{pH}$ (between $\mathrm{pH} 9$ and 12). The silanes (APTES and APDMES), ethanol, sodium chloride $(\mathrm{NaCl})$, sodium hydroxyde $(\mathrm{NaOH})$ and chlorydric acid $(\mathrm{HCl})$, were purchased from Sigma Aldrich.

The deposits were made on silicon substrates purchased from Tracit. ${ }^{45}$ The Milli Q water was obtained with the Direct-Q 3 of Millipore. The $\mathrm{pH}$ of the solution was measured with a $\mathrm{pH}$ meter (Sartorius, PT-10) and an electrode (Sartorius, PY-P22), and ajusted with addition of sodium hydroxyde and chlorydric acid just before measurement.

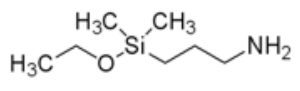

(a) APDMES

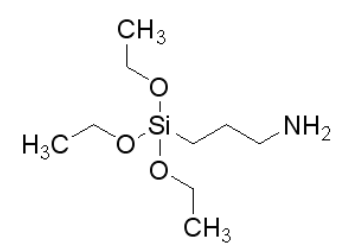

(b) APTES

Figure 2: Molecules used for the silica functionalisation.

\section{Surface functionalisations}

Before being functionalised, the wafers were cleaned by immersion in a piranha solution ( 2 parts $\mathrm{H}_{2} \mathrm{SO}_{4}, 1$ part $\mathrm{H}_{2} \mathrm{O}_{2}$ ) during 25 minutes at $70^{\circ} \mathrm{C}$. Caution: Piranha solution is highly corrosive and extremely reactive with organic substances. Gloves, goggles, laboratory coat, and face shields are needed for protections. Then, the wafer were rinsed in milli Q water then in ethanol before 
silanisation (functionalisation by silane: APDMES, APTES). Solutions were freshly prepared by direct dissolution in ethanol for silanes. The final silanes concentration was $1 \%$. The surfaces were functionalised by immersion in solutions during one night at room temperature. In the silane solution, the molecules were grafted on the substrate (covalent bond). The excess of ungrafted silanes was removed by ultrasonication during 2 minutes in ethanol. The mechanism of self assembled monolayers formation during silanization process is presented by Wasserman et al. ${ }^{41}$

\section{Atomic Force Microscopy}

In order to characterize the surface functionalisation and to apply this functionalisation for microhandling, a commercial atomic force microscope (stand-alone SMENA scanning probe microscope NT-MDT) has been used. The experiment was done in the "Nanorol platform" whose aim is to measure the micromanipulation nanoforce.

\section{AFM Force measurement}

Force measurements were performed in order to characterize the functionalisations. The force measurement performed on this Atomic Force Microscope (AFM) is based on the measurement of the AFM cantilever deformation with a laser deflection sensor (Figure 3). The silicon rectangular AFM cantilever, whose stiffness is $0.3 \mathrm{~N} / \mathrm{m}$, was fixed and the substrate moved vertically. As the applicative objective of this work is to improve reliability of micro-object manipulation, interactions have been studied between a micrometric sphere and a plane. Measurements were in fact performed with a cantilever where a borosilicate sphere $\left(\mathrm{r}_{2}=5 \mu \mathrm{m}\right.$ radius $)$ was glued on the extremity and below this one (Ref.:PT.BORO.SI.10, company Novascan Technologies, Ames, USA). All measurements were done at the driving speed of $200 \mathrm{~nm} / \mathrm{s}$ to avoid the influence of the hydrodynamic drag forces. ${ }^{46}$ For each sample, nine measures were done in different points. The forces standard deviation calculated for nember of nine pull-in and pull-off experiments was less than $10 \%$. 


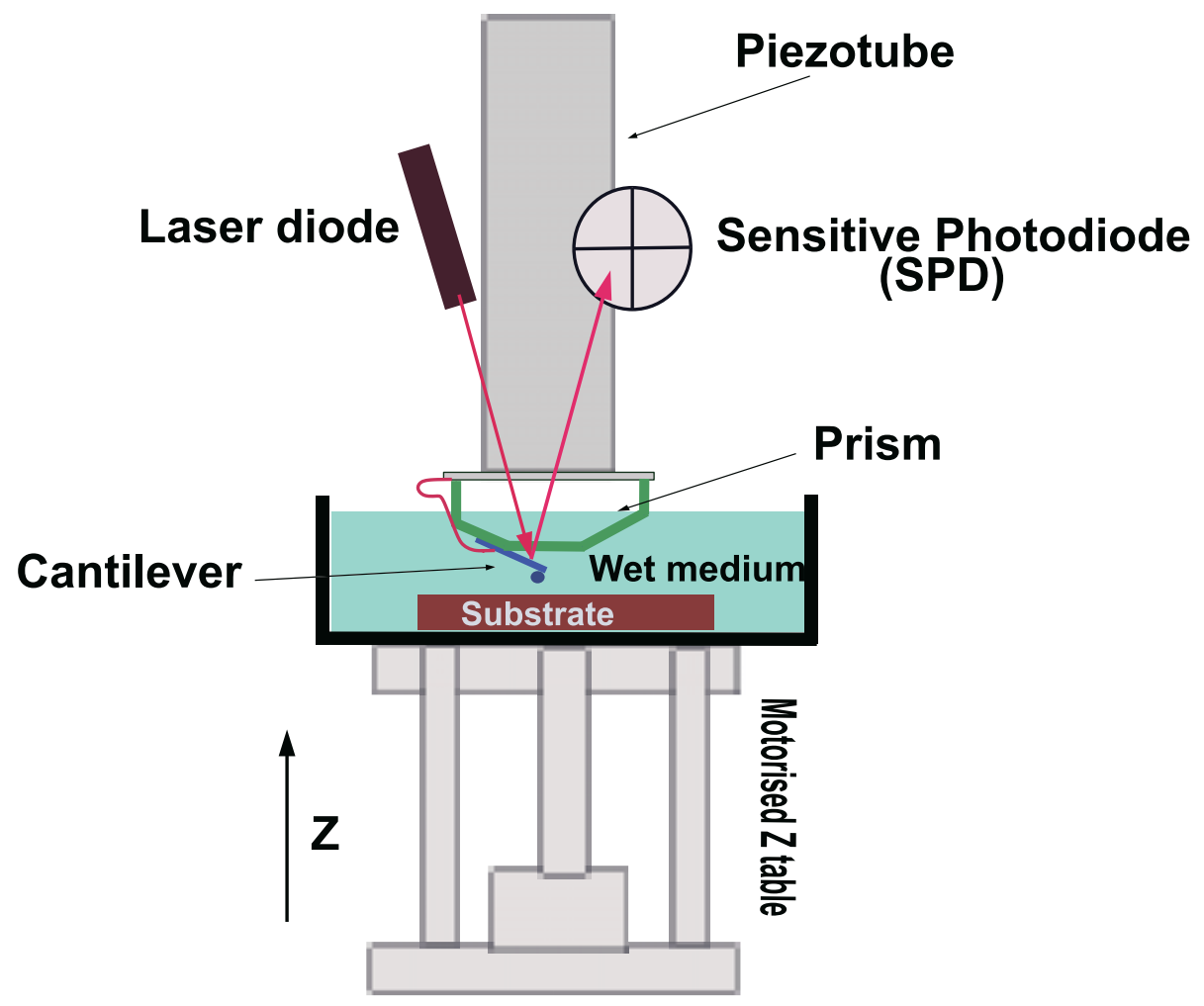

Figure 3: The AFM-related setup used for force measurements. 


\section{Typical distance-forces curves}

The first type of behaviour is presented in Figure 4. In this case, an attractive force (pull-in force) is measured when the sphere is coming close to the substrate (near -20 nN, Figure 4). In Figure 4, we clearly measured a pull-off force which represents the adhesion between the borosilicate sphere on the tip and the functionalised substrate. In this example, the pull-off force is reaching $-1.1 \mu \mathrm{N}$. This behaviour represents an attraction between surfaces.

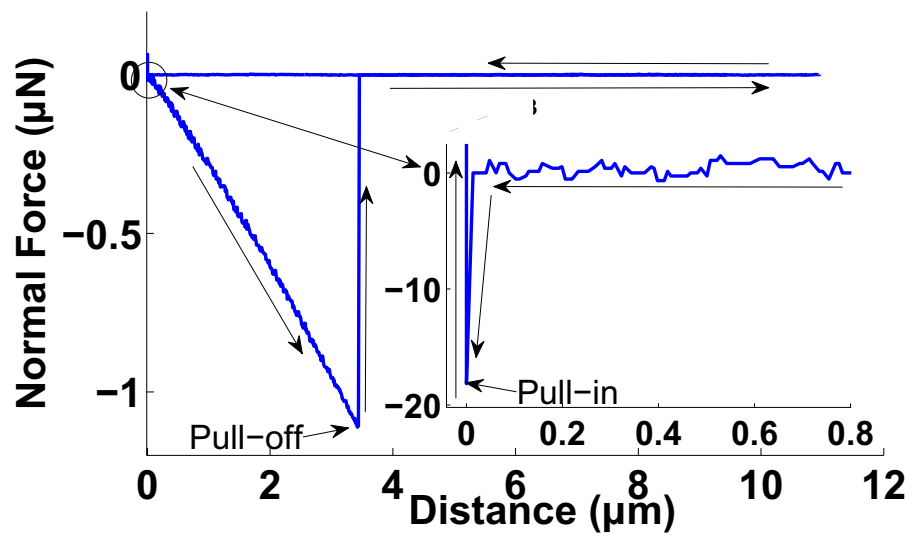

Figure 4: Force-distance curves on functionalised APTES in air medium obtained (spring constant $0.3 \mathrm{~N} / \mathrm{m})$.

The second type of behaviour is presented in Figure 5. In this case, there is repulsion between surfaces. We observe a repulsion (positive pull-in force near $0.75 \mu \mathrm{N}$ ) and no pull-off force between both surfaces. To summarize, a attractive interaction (respectively repulsive) is observed between two surfaces when a pull-in force is negative (respectively positive) and the pull-off forces are always negative.

\section{Micromanipulation}

The aim of this force measurement is to find the conditions in liquid medium where the properties of the grippers or the micro-object are switching in order to facilitate the grasping and the releasing of the microcomponents. In order to verify the adhesion force measurement made by the AFM in the case of micromanipulation, we have done several experiments of pick and place, in different $\mathrm{pH}$ 


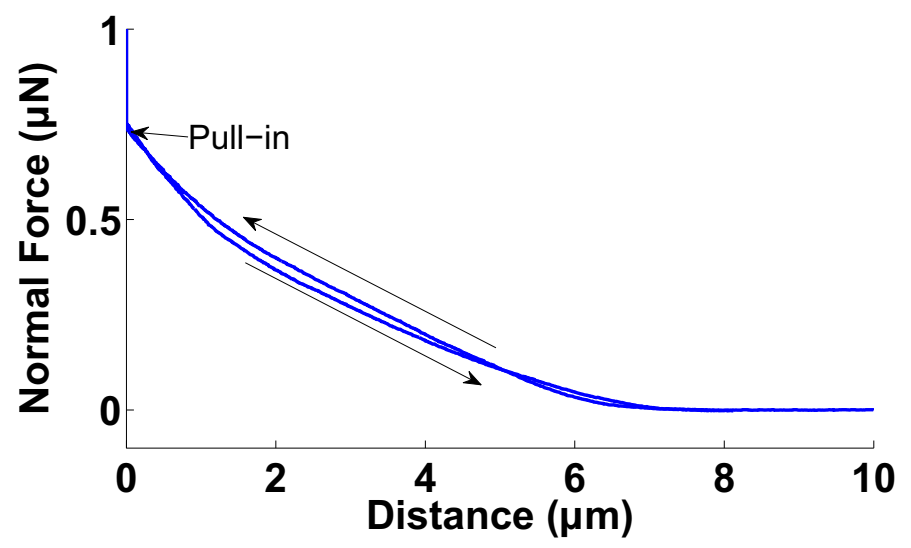

Figure 5: Force-distance curve for the APDMES functionalised substrate in liquid medium obtained (spring constant $0.3 \mathrm{~N} / \mathrm{m}$ ).

solutions. For this, we used a silicon tipless cantilever (Point Probe Technology) functionalised by APTES and glass spheres whose diameter was around 50 micrometers. The spring constant and the resonance frequency at these tips were in the range of $0.02-0.77 \mathrm{~N} / \mathrm{m}$ and $6-21 \mathrm{kHz}$ respectively.

\section{Results and discussion}

\section{Influence of the $\mathrm{pH}$ on the interaction}

Experiments have been done in liquid medium with the functionalised surface and a cantilever grafted with APTES or a non-functionalised cantilever. The $\mathrm{pH}$ of the solution varied by addition of sodium hydroxide or hydrochloric acid. The surface stayed in the solution for 2 minutes before the measurement, in order to equilibrate the system. Force measurement in liquid has been also compared with measurement done in air.

\section{Functionalised surface}

First, the measurements were done with a cantilever and a non-funtionalised sphere. The measure of the pull-in and pull-off forces are presented in Figure 6. 


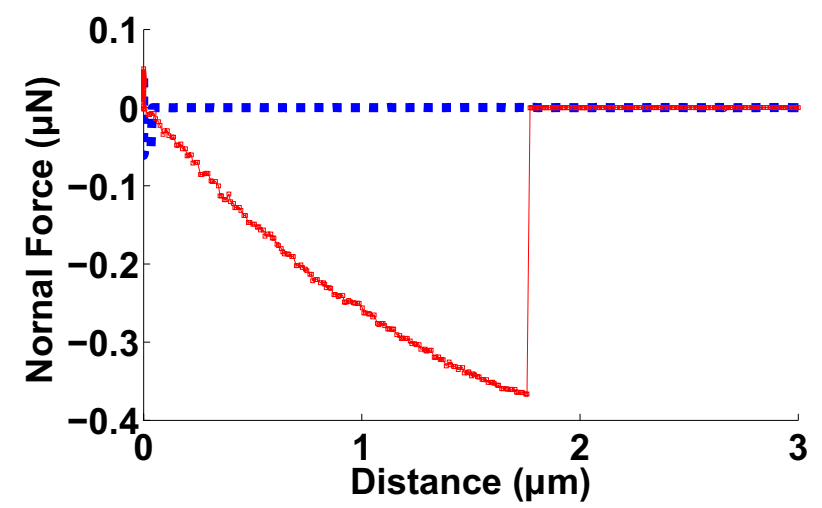

(a)

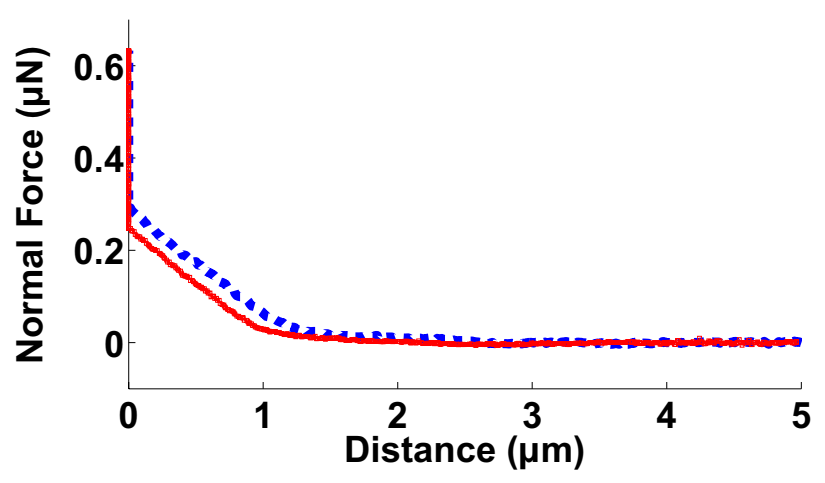

(b)

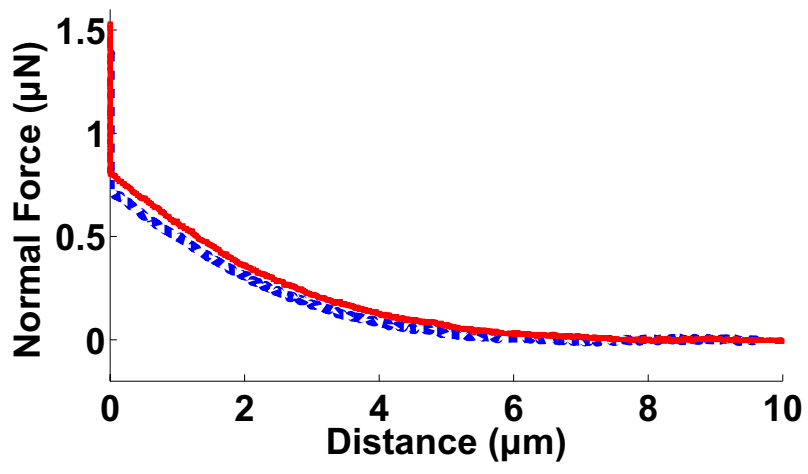

(c)

Figure 6: Force-distance curve for the APTES functionalised substrate in liquid medium at different $\mathrm{pH}$ (spring constant $0.3 \mathrm{~N} / \mathrm{m}$ ): a) $\mathrm{pH}$ natural (near 5.5), b) $\mathrm{pH}$ 9, c) $\mathrm{pH}$ 12. The dash line (blue) are the approach of the surface near the cantilever, and the full line (red) the retract. 
In this figure, we note that the $\mathrm{pH}$ influences significantly the forces between the cantilever and the surface. At natural $\mathrm{pH}$, a attractive pull-in is measured, near $-60 \mathrm{nN}$, with an important pull-off - $350 \mathrm{nN}(6(\mathrm{a}))$. When the $\mathrm{pH}$ increases the pull-in force is inverted and becomes repulsive respectively $280 \mathrm{nN}$ and $770 \mathrm{nN}$ at $\mathrm{pH} 9$ and 12 (6(b), 6(c)). Moreover, the adhesion forces disappear. The average values of the different measurements, (pull-in and pull-off forces), at different $\mathrm{pH}$, are summarized in the Table 1.

Table 1: Influence of the $\mathrm{pH}$ on the pull-in and pull-off forces obtained (spring constant $0.3 \mathrm{~N} / \mathrm{m}$ ) for APTES and APDMES grafted on the surface.

\begin{tabular}{|c|c|c|c|c|}
\cline { 2 - 5 } \multicolumn{1}{c|}{} & \multicolumn{2}{c|}{ APTES } & \multicolumn{2}{c|}{ APDMES } \\
\hline Medium & $\begin{array}{c}\text { Pull-in } \\
(\mathrm{nN})\end{array}$ & $\begin{array}{c}\text { Pull-off } \\
(\mathrm{nN})\end{array}$ & $\begin{array}{c}\text { Pull-in } \\
(\mathrm{nN})\end{array}$ & $\begin{array}{c}\text { Pull-off } \\
(\mathrm{nN})\end{array}$ \\
\hline pH 2 & 0 & $-176 \pm 15$ & 0 & $-93 \pm 14$ \\
\hline pH nat & $-59.5 \pm 8$ & $-387 \pm 38$ & $-29.8 \pm 6$ & $-353 \pm 21$ \\
\hline pH 9 & $282 \pm 31$ & 0 & $377 \pm 49$ & 0 \\
\hline pH 12 & $768 \pm 63$ & 0 & $1100 \pm 130$ & 0 \\
\hline Air & $-13.2 \pm 1.5$ & $-1150 \pm 90$ & $-4.97 \pm 1$ & $-769 \pm 72$ \\
\hline
\end{tabular}

In this table, we observe that the phenomena described above for APTES is the same for APDMES. In fact at natural $\mathrm{pH}$ (near 5.5), the interaction is attractive with an important adhesion force and at basic $\mathrm{pH}$, above 9, the interaction is repulsive. At $\mathrm{pH} 2$, we do not detected pull-in force probably because the charge density on the silica cantilever was too low. In this table, we show that the forces measured with APDMES grafted are lower than APTES. We can explain this by the fact that the quantity of molecules grafted on the substrate is more important for APTES than APDMES.

As the charges on the surface of the silica cantilever are negative or null, the surface density $\sigma$ of APTES and APDMES verifies:

$$
\begin{aligned}
& \text { for } \mathrm{pH} \text { nat or } 2, \sigma \geq 0 \\
& \text { for } \mathrm{pH} 9 \text { or } 12, \sigma \leq 0
\end{aligned}
$$


In fact, at water $\mathrm{pH}$ (near 5.5-6) the positive charges induced by the functionalisation are greater than the negative charges induced by the hydroxyl groups. At this $\mathrm{pH}$ value, the silica was weakly negative. ${ }^{47}$ In basic $\mathrm{pH}$, the negative charges are predominant.

The inversion of the interaction forces during the variation of the $\mathrm{pH}$ of the solution represents a great interest in micromanipulation. The control of the $\mathrm{pH}$ is in fact able to switch from an attractive behaviour (grasping) to a repulsive behaviour (release).

\section{Functionalised surface and cantilever}

Secondly, the cantilever was functionalised with the APTES silane and without sonification step. Similar experiments as previous were performed in aqueous solution of $\mathrm{pH}$ that varied between 2 and 12. The force-distance curves obtained with a APDMES grafted on the substrate are presented in Figure 7.

Contrary to the previous case, the forces measured were always repulsive between the functionalised cantilever with APTES and the APDMES grafted on the surface. We did not detect any pull-off force. There was in fact no adhesion between both functionalised objects. A cantilever deformation was observed on an important distance (typically several microns) when the sphere is approaching from the surface. This distance increases with the value of the repulsion force. This large interaction distance typically is discussed in the following. The average values of the force measurements at different $\mathrm{pH}$, are summarized in the Table 2.

In this table, we note that the $\mathrm{pH}$ of the medium changes the value of the repulsive force between the cantilever and the surface but the behaviour stays always repulsive. For acidic and natural $\mathrm{pH}$, the repulsion can be explained by the positive charges of the aminosilane grafted on the surface. For basic $\mathrm{pH}$, repulsion is induced by the negative charges of the silicon substrate down to the functionalisation. Indeed, from $\mathrm{pH} 9$, the positive charges of the aminosilane are not sufficient 


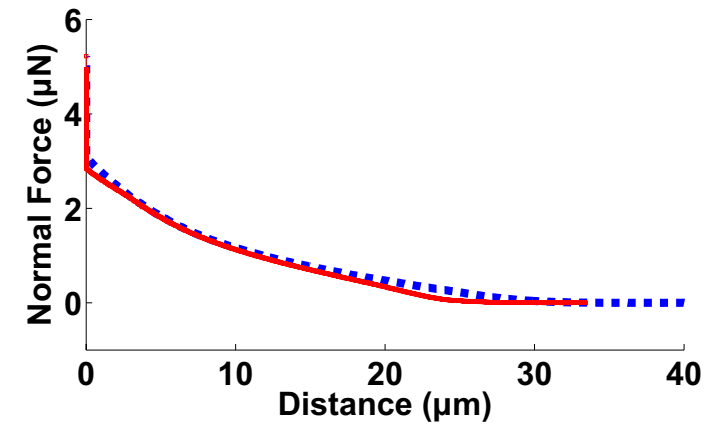

(a)

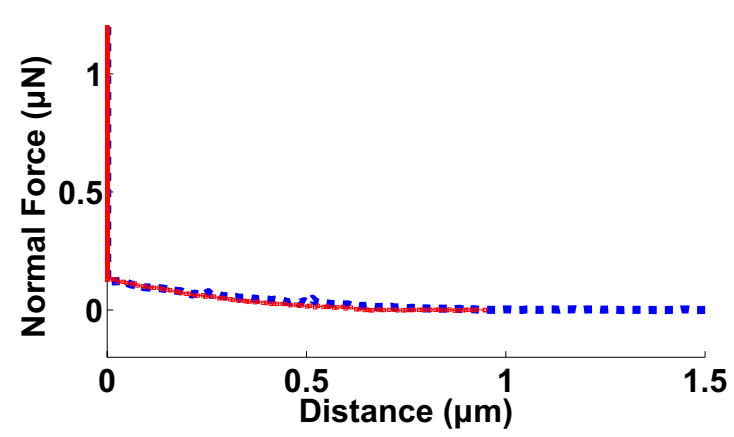

(c)

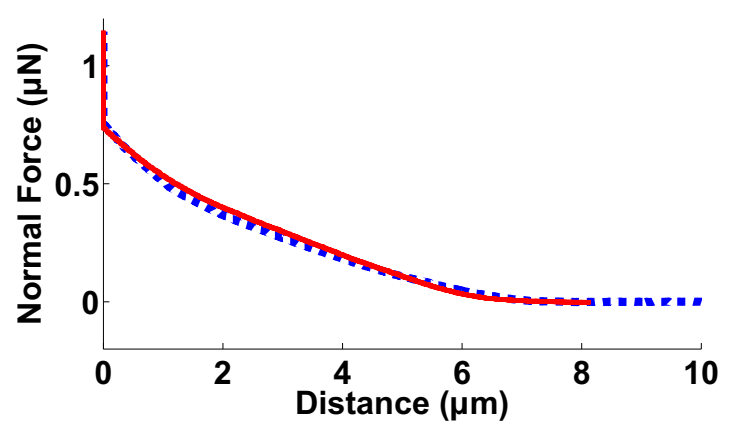

(b)

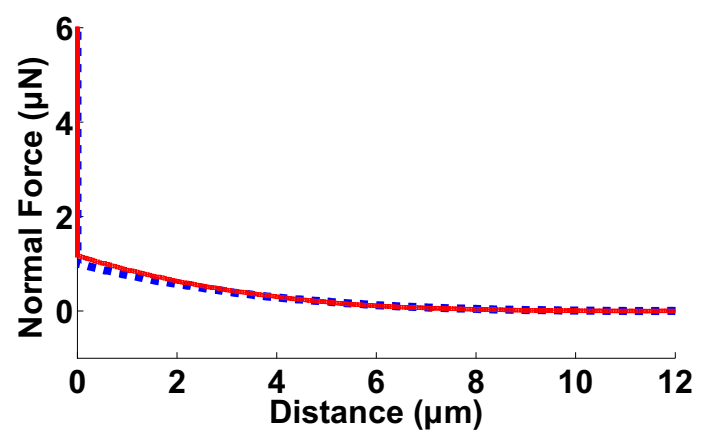

(d)

Figure 7: Force-distance curve for the APTES functionalised substrate and cantilever in liquid medium at different $\mathrm{pH}$ obtained with a tip functionalised APTES (spring constant $0.3 \mathrm{~N} / \mathrm{m}$ ): a) $\mathrm{pH} 2, \mathrm{~b}) \mathrm{pH}$ natural (near 5.5), c) $\mathrm{pH} 9$, d) $\mathrm{pH}$ 12. The dash line (blue) are the approach of the surface near the cantilever, and the full line (red) the retract.

Table 2: Influence of the $\mathrm{pH}$ on the pull-in and pull-off forces $(\mathrm{nN})$ obtained with a tip functionalised APTES (spring constant $0.3 \mathrm{~N} / \mathrm{m}$ ) for APTES and APDMES grafted on the surface.

\begin{tabular}{|c|c|c|c|c|}
\cline { 2 - 5 } \multicolumn{1}{c|}{} & \multicolumn{2}{c|}{ APTES } & \multicolumn{2}{c|}{ APDMES } \\
\hline Medium & $\begin{array}{c}\text { Pull-in } \\
(\mathrm{nN})\end{array}$ & $\begin{array}{c}\text { Pull-off } \\
(\mathrm{nN})\end{array}$ & $\begin{array}{c}\text { Pull-in } \\
(\mathrm{nN})\end{array}$ & $\begin{array}{c}\text { Pull-off } \\
(\mathrm{nN})\end{array}$ \\
\hline pH 2 & $3190 \pm 247$ & 0 & $3080 \pm 223$ & 0 \\
\hline pH nat & $655 \pm 50$ & 0 & $735 \pm 60$ & 0 \\
\hline pH 9 & $150 \pm 13$ & 0 & $114 \pm 15$ & 0 \\
\hline pH 12 & $983 \pm 62$ & 0 & $989 \pm 66$ & 0 \\
\hline Air & 0 & $-91 \pm 23$ & 0 & $-136 \pm 35$ \\
\hline
\end{tabular}


to totally screening the negative charges of the silicon. However at $\mathrm{pH} 9$, the charge screening induced by some $\mathrm{NH}_{3}^{+}$explains why the repulsions are lower with a functionalised cantilever $(\mathrm{pH}$ 9 in Table 2) than with a non-functionalised cantilever ( $\mathrm{pH} 9$ in Table 1). Moreover, at $\mathrm{pH} 12$, behaviours of the functionalised surface and the non-functionalised surface are quite similar. In fact, the aminosilane has any positive charges left and the repulsion is only induced by the negative charges on silicon and borosilicate. However the unstability of the silane layer in basic solutions was already measured by Wasserman. ${ }^{41}$ The monolayer get immediately deteriorated on exposing to aqueous base and a complete removal of monolayer was observed within $60 \mathrm{~min}$. The destruction of monolayer in the high basic solution $(\mathrm{pH} 12)$ is due to the hydrolysis of Si-O-Si bonds. ${ }^{41}$ The stability range depends on the silane molecules and on the subatrate where it is grafted. In our case, the destruction of the silane layer is observed at $\mathrm{pH} 12$ by the gradued decreasing of the AFM laser-photodiode reading during force-distance measurement. Instead, we noticed no modification of the laser-photodiode value for $\mathrm{pH} 9$ and the subsequent measurements, repeated three day after, were the same. So, the silane layer at $\mathrm{pH} 9$ is stable. Also, when the cantilever was immerged in acid solution at room temperature, the laser-photodiode value stayed identical during the measurement, demonstrating the silane layer stability to acid $\mathrm{pH}$. Lenfant ${ }^{43}$ has already demonstrated the stability of the silane layer for a long period (up to a couple of days) at $\mathrm{pH} 1$.

In micromanipulation, the repulsion between two objects is an interesting behaviour in order to make easier the separation of two objects, whatever the $\mathrm{pH}$ of the solution. Indeed, the release of micro-objects will be easier if both micro-object and gripper are functionalised with aminosilane which induces repulsive force.

\section{Modeling of the surface charges}

\section{Multiscale modeling}

The range of the force measured is clearly greater than the current works done in electrical double layer analysis, where the typical interaction distance is about several tens of nanometers. ${ }^{48-50}$ 
These studies present experiments and models of the coupling between the surface charges, the interaction distance and the electrical surface potential for interaction distance lower than the micron. In our case, the interaction lengths are greater than the micron and cannot be modeled by these approaches.

In the literature, some works deal with high long range interaction on polymer surfaces. ${ }^{51,52}$ Interaction distance has the same order of magnitude as our works, but the magnitude of forces was not measured. Explanations provided by the authors are based on thousands layers of tightly bound water. We are proposing an other explanation based on multi-scale analysis:

In the nanoscale or in a chemical point of view, the distance between both surfaces greater than 1 micrometer could be considered as infinite. Each surface can be thus considered individually. Chemical interaction with the electrolyte induces an electrical charge density on the surface. We will show that the order of magnitude of this charge density is comparable to those predict in the case of double layer analysis.

In the microscale, both surfaces can be considered as an uniformly charged surface whose charges are constant. The liquid medium can be modeled by a dielectric. Interaction forces can be deduced by Coulomb law between both surfaces. We will show that the interaction distance of several microns can be easily explained by Coulomb forces.

\section{Model of the Coulomb force}

In order to determine an analytic relationship between the Coulomb force and the surface density on both surfaces, we assume that the surface is large enough to be considered as infinite compared to the sphere whose radius is $r_{2}=5 \mu \mathrm{m}$. The electric field $E_{1}$ induced by the surface charge density $\sigma_{1}$ of the substrate is uniform:

$$
\overrightarrow{E_{1}}=\frac{\sigma_{1}}{2 \varepsilon_{3} \varepsilon_{0}} \overrightarrow{n_{1}}
$$


where $\varepsilon_{0}$ is the electric permittivity of the vacuum and $\varepsilon_{3}$ the relative permittivity of the medium (for water, $\varepsilon_{3}=80$ ), $\overrightarrow{n_{1}}$ unit vector perpendicular to the substrate. The repulsive Coulomb force applied by the gripper on the object whose charge is $q_{2}$ is thus:

$$
\vec{F}_{\text {pull-in }}=q_{2} \cdot \vec{E}_{1}=2 \pi r_{2}^{2} \frac{\sigma_{1} \sigma_{2}}{\varepsilon_{3} \varepsilon_{0}} \overrightarrow{n_{1}}
$$

where $\sigma_{2}$ is the charge density on the sphere whose radius is $r_{2}$.

If both objects have the same surface density $\sigma_{1}$, this later can be deduced from the force measurement:

$$
\left|\sigma_{1}\right|=\left(F_{\text {pull-in } 1-1} \frac{\varepsilon_{3} \varepsilon_{0}}{2 \pi r_{2}^{2}}\right)^{\frac{1}{2}}
$$

The sign of $\sigma_{1}$ should be determined by considering the chemical functions (equations (1)).

Moreover, in case of an interaction between two different functionalised surfaces, the charge density $\sigma_{2}$ of the second surface is done by:

$$
\sigma_{2}=\frac{F_{p u l l-i n 1-2}}{\sigma_{1}} \frac{\varepsilon_{3} \varepsilon_{0}}{2 \pi r_{2}^{2}}
$$

\section{Identification of the surface charges}

In the following, we assume that the surface density on APTES grafted on borosilicate and on silicon are identical. The equation (4) has been used to determine the charge density of APTES (see in Table 3). The equation (5) has been used to determine the electrical surface density of APDMES (see in Table 3).

In this table, the sign of the charge density was determined according to the equations (1). Buron et al. are also found a positive charge density at $\mathrm{pH}$ natural (5.5). ${ }^{53}$ The value of the charge density corresponds to a double layer potential near $50 \mathrm{mV}$ at $\mathrm{pH} 2$. This potential value is widely found for surfaces deposited with polymers or silanes. ${ }^{54-56}$ In the case of a monolayer, it is generally found that the electrokinetic potential after adsorption has the same sign as the adsorbed 
Table 3: Electrical surface density of the functionalised surface in function of the $\mathrm{pH}$

\begin{tabular}{|c|c|c|}
\cline { 2 - 3 } \multicolumn{1}{c|}{} & APTES & APDMES \\
\hline pH & $\sigma_{1}\left(\mu \mathrm{C} / \mathrm{cm}^{2}\right)$ & $\sigma_{2}\left(\mu \mathrm{C} / \mathrm{cm}^{2}\right)$ \\
\hline pH 2 & $+0,38$ & $+0,36$ \\
\hline pH nat & $+0,17$ & $+0,19$ \\
\hline pH 9 & $-0,08$ & $-0,06$ \\
\hline pH 12 & $-0,21$ & $-0,21$ \\
\hline
\end{tabular}

polyelectrolyte, which means that there is an excess of polymer charge density in comparison with the structural surface charge. Using the Gouy-Chapman relationship for diffuse layer charge density, ${ }^{57}$ the charge excess corresponding to a potential around $50 \mathrm{mV}$ can be calculated. Theoretical studies carried out by Cohen-Stuart et al. ${ }^{56}$ and experimental illustrations ${ }^{55}$ have shown that once the surface potential reaches a given value, an electrostatic repulsion is created in the interface that prevents additional molecules from reaching the substrate. In other words, polyelectrolyte adsorption is kinetically limited, with a threshold for surface saturation when the zeta potential reaches about $\pm 40 \mathrm{mV}$. This all applies well to the present measurements.

\section{Interaction distance modeling}

This section shows that Coulomb law between two surfaces whose surface charges are constant, are able to induce high long range interactions. A Finite Element Model (FEM) of the Coulomb force between a finite surface and a sphere with an identical surface charges $\sigma_{1}$ (see in Table 3) has been simulated with the software COMSOL Multiphysics 3.5. Comparative results between experiments and simulated forces are presented in the Figure 8. It clearly shows that the model using Coulomb force between two charged surfaces is able to explain both the high long range of the interaction and the level of force.

\section{Application of functionalised surfaces in micromanipulation}

The behaviour described in Table 1 shows a transition between attraction in natural $\mathrm{pH}$ and repulsion in $\mathrm{pH}$ 9. This switching behaviour can be used to control the grasping and the release of a 


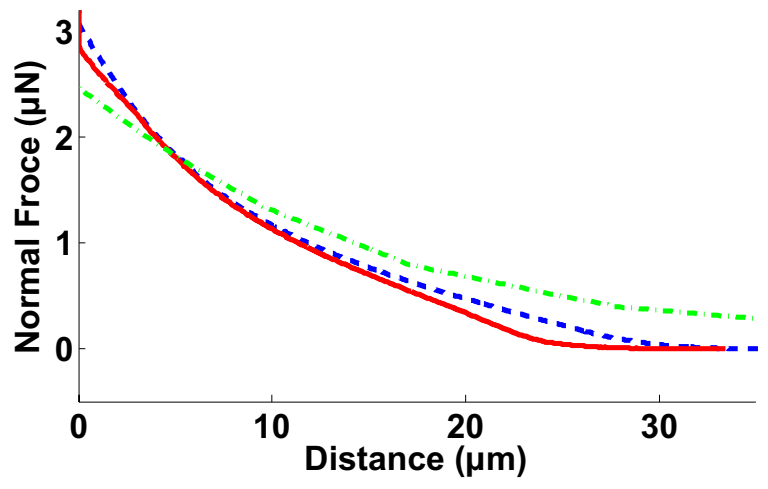

Figure 8: Experimental and simulated Force-distance curve for the APTES functionalised substrate and cantilever at $\mathrm{pH}$ 2. The dash line (blue) is the experimental approach of the surface, the full line (red) is the experimental retract and the dash-dot line (green) is the simulated Coulomb force.

micro-object manipulated with a microgripper. Two scenarii can be presented (see in Figure 9). In the first case, grasping and release occur in two different medium in order to guarantee adhesion during the grasping and repulsion during the release. In the second case, the $\mathrm{pH}$ of the medium has to be changed during manipulation. A microfluidic system could be build to induce laminar flow of acidic or basic solution sequentially in the manipulation range. The laminar flow should be able to switch rapidly the $\mathrm{pH}$ without disturbing the position of the object on the substrate.

This microhandling method has been first tested on AFM. The Figure 10 shows first experiments made with the AFM with a tipless cantilever functionalised with APTES and a free functionalisation glass sphere. At natural $\mathrm{pH}$ a glass sphere, whose diameter is around 50 micrometers, is 'grasped' with the tipless cantilever (Figure 10(a)). For this, the cantilever was lowered down to the sphere and a force of $20 \mu \mathrm{N}$ was applied. When the cantilever went up the borosilicate sphere stayed attached to the cantilever thanks to the attractive force (natural $\mathrm{pH}$ ) and the adhesion force which are near $60 \mathrm{nN}$ and $387 \mathrm{nN}$ respectively (Table 1). Afterwards, the $\mathrm{pH}$ was increased by addition of $\mathrm{NaOH}$ solution. The behaviour was inverted and when $\mathrm{pH}$ was near 9 , the sphere has been released (Figure 10(b)). Indeed, the attractive and adhesion force disappeared and an repulsive force of $282 \mathrm{nN}$ appeared between the cantilever and the sphere (Table 1). 


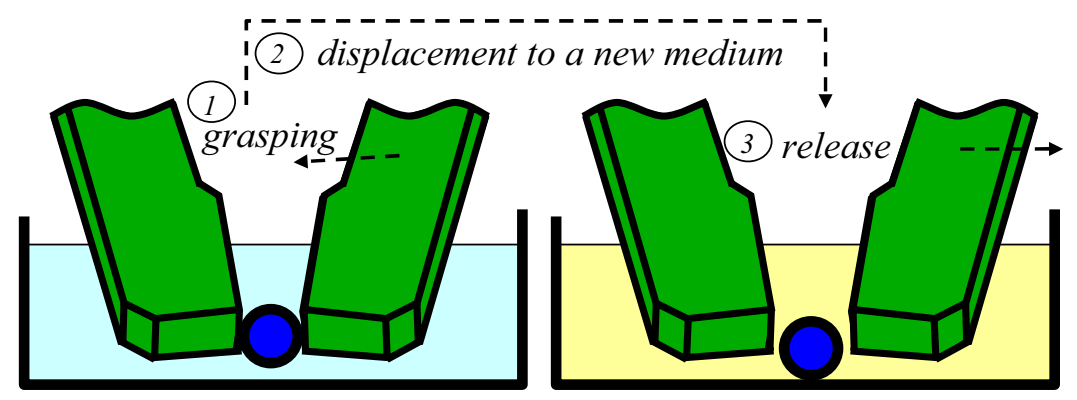

(a) first scenario

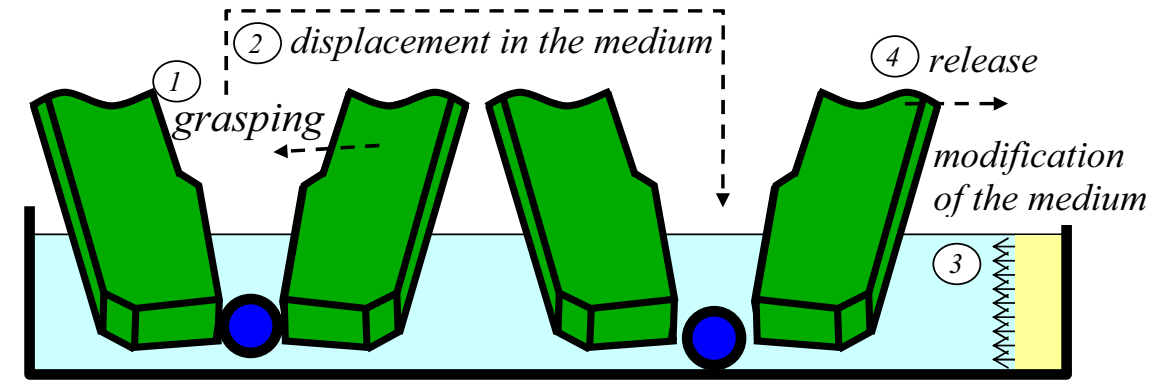

(b) second scenario

Figure 9: Scenarii of robotic microhandling coupling with chemical functionnalisation

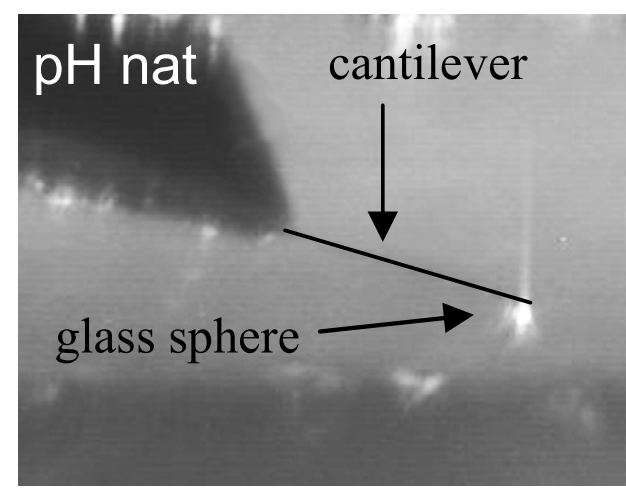

(a)

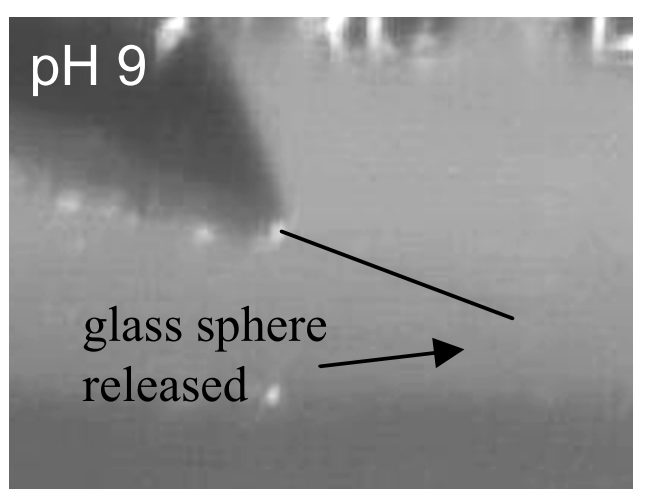

(b)

Figure 10: Grasp at natural $\mathrm{pH}$ (a) and Release at $\mathrm{pH} 9$ of the sphere with functionalised cantilever 


\section{Conclusions}

In this paper, we have studied interaction behaviour between two functionalised surfaces and between functionalised and neutral surfaces. The experiments were done in function of the $\mathrm{pH}$ of liquid and compared with air medium. The substrates were functionalised by two chemical compounds using silanisation (grafted of silane molecules). This technic was hold because of the important stability of the silane layer as thermal stability up to $350^{\circ} \mathrm{C}$, chemical stability under different etchants, enduranceas. We have shown that the functionalisation and the $\mathrm{pH}$ of the medium could highly change the adhesion properties. The micro-assembly could be facilitated by a judicious choice of the media and of the functionalisation of both grippers and micro-objects. We have shown that the $\mathrm{pH}$ can be used to control the release of a non-functionalised object during micromanipulations. Furthermore, the use of functionalised grippers and objects enables to simply cancel adhesion on micro-objects. As adhesion is the current highest disturbance in micromanipulation, functionalisation is a promising way to improve micro-object manipulation in the future. This paper consists in a proof of concept of a new promising micromanipulation method. The complet characterisation of this method based on repeatability measurements as well as reliability determination has to be performed. Future works will also focus on the implementation of this method which is able to cancel adhesion perturbations on two-fingered microgripper. Large blocking force required in microassembly will be thus possible.

\section{Acknowledgement}

This work was supported by the EU under HYDROMEL contract NMP2-CT-2006-026622 : Hybrid ultra precision manufacturing process based on positional- and self-assembly for complex micro-products, and by the French National Agency (ANR) under NANOROL contract ANR07-ROBO-0003: Nanoanalyse for micromanipulate. We also acknowledge the support of David Rostoucher and Alex Ivan for their English correction. 


\section{Supporting Information Available}

The "nanorol platform" can be used by external person. The availability and the booking of the station is consultable via internet at: http://nanorol.cnrs.fr/events.php."

\section{References}

(1) Tamadazte, B.; Dembélé, S.; Le Fort-Piat, N. Sensors and Transducers Journal 2009, 5, 3752.

(2) Lambert, P. Capillary Forces in Micro-assembly; Springer, 2008.

(3) Gauthier, M.; Régnier, S.; Rougeot, P.; Chaillet, N. Journal of Micromechatronics 2006, 3, 389-413.

(4) Zhou, Q.; Chang, B.; Koivo, H. N. Ambient environment effects in micro/nano handling. Proc. of the Int. Workshop on Microfactories, Shangai, China, 2004; pp 146-51.

(5) Sariola, V.; Zhou, Q.; Koivo, H. N. Journal of Micro - Nano Mechatronics 2008, 4, 5-16.

(6) Lambert, P. Journal of Micromechanical Microengineering. 2006, 16, 1267-1276.

(7) Dholakia, K.; Reece, P.; Gu, M. Chem. Soc. Rev. 2008, 37, 42-55.

(8) Hunt, T. P.; Westervelt, R. M. Biomedical Microdevices 2006, 8, 227-230.

(9) Arai, F.; Andou, D.; Nonoda, Y.; Fukuda, T.; Iwata, H.; Itoigawa, K. IEEE/ASME Transactions on Mechatronics 1998, 3, 17-23.

(10) Hériban, D.; Gauthier, M. Robotic Micro-assembly of Microparts Using a Piezogripper. Proc. of the 2008 IEEE/RSJ International Conference on Intelligent Robots and Systems, Nice, France, 2008; pp 4042-4047.

(11) Lambert, P.; Régnier, S. International Journal of Micromechatronics 2006, 3, 123-157. 
(12) Driesen, W.; Varidel, T.; Régnier, S.; Breguet, J. Journal of Micromechanics and Microengineering 2005, 15, 259-267.

(13) Gauthier, M.; Gibeau, E.; Hériban, D. Submerged Robotic Micromanipulation and Dielectrophoretic Micro-object Release. Proc. of the IEEE ICARCV 2006 conference, Singapour, 2006.

(14) Dafflon, M.; Lorent, B.; Clavel, R. A micromanipulation setup for comparative tests of microgrippers. International Symposium on Robotics (ISR), 2006.

(15) Dejeu, J.; Rougeot, P.; Gauthier, M.; Boireau, W. accepted in Micronanoletters 2009, 4, 7479.

(16) Wang, T.; Canetta, E.; Weerakkody, T. G.; Keddie, J. L. ACS Applied Materials \& Interfaces 2009, 1, 631-639.

(17) Gong, H.; Garcia-Turiel, J.; Vasilev, K.; Vinogradova, O. I. Langmuir 2005, 21, 7545-7550.

(18) Delettre, A.; Gauthier, M.; Rougeot, P.; Régnier, S. Submitted in Applied Surface Science 2009.

(19) Blomberg, E.; Poptoshev, E.; Claesson, P. M.; Caruso, F. Langmuir 2004, 20, 5432-5438.

(20) Charrault, E.; Gauthier, C.; Marie, P.; Schirrer, R. Langmuir 2009, 25, 5847-5854.

(21) Rabenorosoa, K.; Clevy, C.; Lutz, P.; Gauthier, M.; Rougeot, P. submitted in Micro and Nano Letters 2009.

(22) Vajpayee, S.; Hui, C.-Y.; Jagota, A. Langmuir 2008, 24, 9401-9409.

(23) Murphy, M. P.; Kim, S.; Sitti, M. ACS Applied Materials \& Interfaces 2009, 1, 849-855.

(24) Dejeu, J.; Lakard, B.; Fievet, P.; Lakard, S. Journal of Colloid and Interface Science 2009, in press, year. 
(25) Nolte, A. J.; Chung, J. Y.; Walker, M. L.; ; Stafford, C. M. ACS Applied Materials \& Interfaces 2009, 1, 373-380.

(26) Yeo, C.; Lee, S.; Polycarpou, A. Review of Scientific Instruments 2008, 79, 015111-1 015111-8.

(27) Tormoen, G.; Drelich, J. Journal of Adhesion Science and Technology 2005, 19, 181-198.

(28) Cleaver, J.; Tyrrell, J. Kona 2004, 22, 9-22.

(29) Tambe, N.; Bhushan, B. Nanotechnology 2004, 15, 1561-1570.

(30) Heim, L.; Blum, J. Physical Review Letters 1999, 83, 3328-3331.

(31) Haiat, G.; Barthel, E. Langmuir 2007, 23, 11643-11650.

(32) Xu, L.; Vadillo-Rodriguez, V.; Logan, B. Langmuir 2005, 21, 7491-7500.

(33) Sigmund, W.; Sindel, J.; Aldinger, F. Progress in Colloid and Polymer Science 1997, 105, $23-26$.

(34) Bowen, W.; Doneva, T.; Austin, J.; Stoton, G. Colloids and Surfaces A 2002, 201, 73-83.

(35) G. Decher, J. S. Sequential Assembly of Nanocomposite Materials; Wiley-VCH, 2003.

(36) Demirel, G.; Caglayan, M. O.; Garipcan, B.; Piskin, E. Surface Science 2008, 602, 952-959.

(37) Maas, J.; Stuart, M. C.; Sieval, A.; Zuilhof, H.; Sudhölterb, E. Thin Solid Films 2003, 426, $135-139$.

(38) Mangeat, T.; Berthier, A.; Elie-Caille, C.; Perrin, M.; Boireau, W.; Pieralli, C.; Wacogne, B. Laser Physics 2009, 19, 1-7.

(39) Moreau, O.; Portella, C.; Massicot, F.; Herry, J.; Riquet, A. Surface \& Coatings Technology 2007, 201, 5994-6004. 
(40) Wang, J.; Guo, D.-J.; Xia, B.; Chao, J.; Xiao, S.-J. Colloids and Surface A : Physicochemical and Engineering Aspects 2007, 305, 66-75.

(41) Wasserman, S.; Tao, Y.-T.; Whitesides, G. Langmuir 1989, 5, 1074-1087.

(42) Ulman, A. An Introduction to Ultrathin Organic Films From Langmuir-Blogett to SelfAssembly; Academic Press, 1991.

(43) Lenfant, S. Ph.D. thesis, University of Science and Technology, Lille, France, 2001.

(44) Dove, P. M.; Craven, C. M. Geochimica et Cosmochimica Acta 2005, 69, 4963Ú4970.

(45) Agnus, J.; Hériban, D.; Gauthier, M.; Pétrini, V. Precision Engineering 2009, In Press, Corrected Proof, Available online 28 February 2009.

(46) Vinogradova, O. I.; Yakubov, G. E. Langmuir 2003, 19, 1227-1234.

(47) Foissy, A.; Persello, J. The Surface Properties of Silicas; John Wiley \& Sons, 1998.

(48) Adamson, A. W.; Gast, A. P. Physical Chemistry of Surface, 6th ed.; Wiley Intescience Publication, 2002.

(49) Briscoea, W. H.; Attardb, P. Journal of Chemical Physics 2002, 11, year.

(50) Attard, P. Adv. Chem. Phys. 1996, 92, 1-159.

(51) Zheng, J.; Pollack, G. H. In Water and the Cell; Gerald H. Pollack, I. L. C., Wheatley, D. N., Eds.; Springer Netherlands, 2006; Chapter 8Solute Exclusion and Potential Distribution Near Hydrophilic Surfaces, pp 165-74.

(52) Zheng, J.-M.; Pollack, G. H. Physical review. E, Statistical, nonlinear, and soft matter physics 2003, 68, 031408.1-031408.7.

(53) Buron, C.; Filiâtre, C.; Membrey, F.; Bainier, C.; Charraut, D.; Foissy, A. Colloids and Surface A : Physicochemical and Engineering Aspects 2007, 305, 105-111. 
(54) Dejeu, J.; Buisson, L.; aand Cyrille Roidor, M. C. G.; Membrey, F.; Charraut, D.; Foissy, A. Colloids and Surfaces A: Physicochemical and Engineering Aspects 2006, 288, 26.

(55) Geffroy, C.; Labeau, M.; Wong, K.; Cabane, B.; Stuart, M. C. Colloids and Surfaces A: Physicochemical and Engineering 2000, 172, 47.

(56) Cohen-Stuart, M.; Hoogendam, C.; de Keizer, A. Journal of Physics: Condensed Matter. 1997, 9, 7767.

(57) Hunter, R. Introduction to Modern Colloid Science; Oxford University Press, 1993.

\section{List of Figures}

1 Principle of the Robotic Microhandling controlled by Chemical Self Assembly Monolayer $(\mathrm{SAM}) \ldots \ldots \ldots \ldots \ldots \ldots$

2 Molecules used for the silica functionalisation. . . . . . . . . . . . . 6

3 The AFM-related setup used for force measurements. . . . . . . . . . . . . . 8

4 Force-distance curves on functionalised APTES in air medium obtained (spring constant $0.3 \mathrm{~N} / \mathrm{m}) \ldots \ldots \ldots \ldots \ldots \ldots \ldots$

5 Force-distance curve for the APDMES functionalised substrate in liquid medium obtained $($ spring constant $0.3 \mathrm{~N} / \mathrm{m}) . \ldots \ldots \ldots \ldots$

6 Force-distance curve for the APTES functionalised substrate in liquid medium at different $\mathrm{pH}$ (spring constant0.3 N/m): a) pH natural (near 5.5), b) $\mathrm{pH}$ 9, c) $\mathrm{pH} 12$. The dash line (blue) are the approach of the surface near the cantilever, and the full line $($ red $)$ the retract. . . . . . . . . . . . . . . . . . 11 
7 Force-distance curve for the APTES functionalised substrate and cantilever in liquid medium at different $\mathrm{pH}$ obtained with a tip functionalised APTES (spring constant $0.3 \mathrm{~N} / \mathrm{m}$ ): a) $\mathrm{pH} \mathrm{2,} \mathrm{b)} \mathrm{pH}$ natural (near 5.5), c) pH 9, d) $\mathrm{pH}$ 12. The dash line (blue) are the approach of the surface near the cantilever, and the full line (red) the retract. . . . . . . . . . . . . . . . . . . . 14

8 Experimental and simulated Force-distance curve for the APTES functionalised substrate and cantilever at $\mathrm{pH}$ 2. The dash line (blue) is the experimental approach of the surface, the full line (red) is the experimental retract and the dash-dot line (green) is the simulated Coulomb force. . . . . . . . . . . . . . . . . . . 19

9 Scenarii of robotic microhandling coupling with chemical functionnalisation . . . . 20

10 Grasp at natural $\mathrm{pH}$ (a) and Release at $\mathrm{pH} 9$ of the sphere with functionalised can-

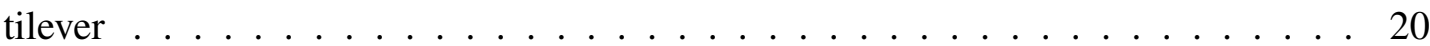

